\title{
BMJ Open Process evaluation protocol for a cluster randomised trial of a complex, nurse-led intervention to improve hypertension management in India
}

\author{
Nikhil Srinivasapura Venkateshmurthy, ${ }^{\oplus 1,2}$ Kevin Mc Namara, ${ }^{3}$ Harriet Koorts, ${ }^{1}$ \\ Sailesh Mohan, ${ }^{2}$ Vamadevan S Ajay, ${ }^{4}$ Devraj Jindal, ${ }^{4}$ Bhaskara Rao Malipeddi, ${ }^{5}$ \\ Ambuj Roy, ${ }^{6}$ Nikhil Tandon, ${ }^{7}$ Dorairaj Prabhakaran, ${ }^{2}$ Tony Worsley, ${ }^{1}$ \\ Ralph Maddison, ${ }^{1}$ Sharleen O'Reilly ${ }^{1}$
}

To cite: Srinivasapura Venkateshmurthy N, Mc Namara $\mathrm{K}$, Koorts $\mathrm{H}$, et al. Process evaluation protocol for a cluster randomised trial of a complex, nurse-led intervention to improve hypertension management in India. BMJ Open 2019;9:e027841. doi:10.1136/ bmjopen-2018-027841

- Prepublication history for this paper is available online. To view these files, please visit the journal online (http://dx.doi org/10.1136/bmjopen-2018027841).

Received 13 November 2018 Revised 16 February 2019 Accepted 13 March 2019

Check for updates

(C) Author(s) (or their employer(s)) 2019. Re-use permitted under CC BY-NC. No commercial re-use. See rights and permissions. Published by BMJ.

For numbered affiliations see end of article.

Correspondence to Dr Nikhil Srinivasapura Venkateshmurthy; nnikhils@deakin.edu.au; nikhil.sv@phfi.org

\section{ABSTRACT}

Introduction India has high prevalence of hypertension but low awareness, treatment and control rate. A cluster randomised trial entitled ' $m$-Power Heart Project' is being implemented to test the effectiveness of a nurse care coordinator (NCC) led complex intervention to address uncontrolled hypertension in the community health centres (CHCs). The trial's process evaluation will assess the fidelity and quality of implementation, clarify the causal mechanisms and identify the contextual factors associated with variation in the outcomes. The trial will use a theory-based mixed-methods process evaluation, guided by the Consolidated Framework for Implementation Research.

Methods and analysis The process evaluation will be conducted in the CHCs of Visakhapatnam (southern India). The key stakeholders involved in the intervention development and implementation will be included as participants. In-depth interviews will be conducted with intervention developers, doctors, NCCs and health department officials and focus groups with patients and their caregivers. NCC training will be evaluated using Kirkpatrick's model for training evaluation. Key process evaluation indicators (number of patients recruited and retained; concordance between the treatment plans generated by the electronic decision support system and treatment prescribed by the doctor and so on) will be assessed. Fidelity will be assessed using Borrelli et afs framework. Qualitative data will be analysed using the template analysis technique. Quantitative data will be summarised as medians (IQR), means (SD) and proportions as appropriate. Mixed-methods analysis will be conducted to assess if the variation in the mean reduction of systolic blood pressure between the intervention CHCs is influenced by patient satisfaction, training outcome, attitude of doctors, patients and NCCs about the intervention, process indicators etc.

Ethics and dissemination Ethical approval for this study was obtained from the ethics committees at Public Health Foundation of India and Deakin University. Findings will be disseminated via peer-reviewed publications, national and international conference presentations.

Trial registration number NCT03164317; Pre-results.
Strengths and limitations of this study

- Theory-based process evaluation, using an evidence-based framework designed to evaluate healthcare innovations.

- The process evaluation is comprehensive, that is, it involves the intervention developers, implementers (the nurse care coordinators [NCCs]), the end users (doctors and patients) and health system actors.

- Process evaluation is being conducted alongside the intervention implementation thus giving information about the early implementation phase when challenges often occur.

- Recruitment of NCCs as key informants may introduce response bias which is planned to be minimised by reassuring the NCCs that the information they provide is only going to strengthen the implementation and has no effect on their employment.

\section{INTRODUCTION}

Non-communicable diseases account for over $60 \%$ of all deaths in India with cardiovascular disease being the leading cause. ${ }^{12}$ Among Indians, cardiovascular disease occurs a decade earlier, is more severe and is associated with a higher rate of mortality compared with their western counterparts. ${ }^{3}$ High systolic blood pressure $(\geq 140 \mathrm{~mm} \mathrm{Hg})$ is the leading metabolic risk factor for cardiovascular disease in India. ${ }^{4}$ The reported prevalence of hypertension in India is $25 \% .^{5}$ This high prevalence is compounded by low levels of awareness, treatment, control and suboptimal self-management. ${ }^{6}$

\section{m-Power Heart Project}

The 'm-Power Heart Project' (hereafter referred to as m-Power) is a cluster randomised controlled trial (RCT) to determine the effectiveness of a nurse care coordinator (NCC) led, mobile health (mHealth) 


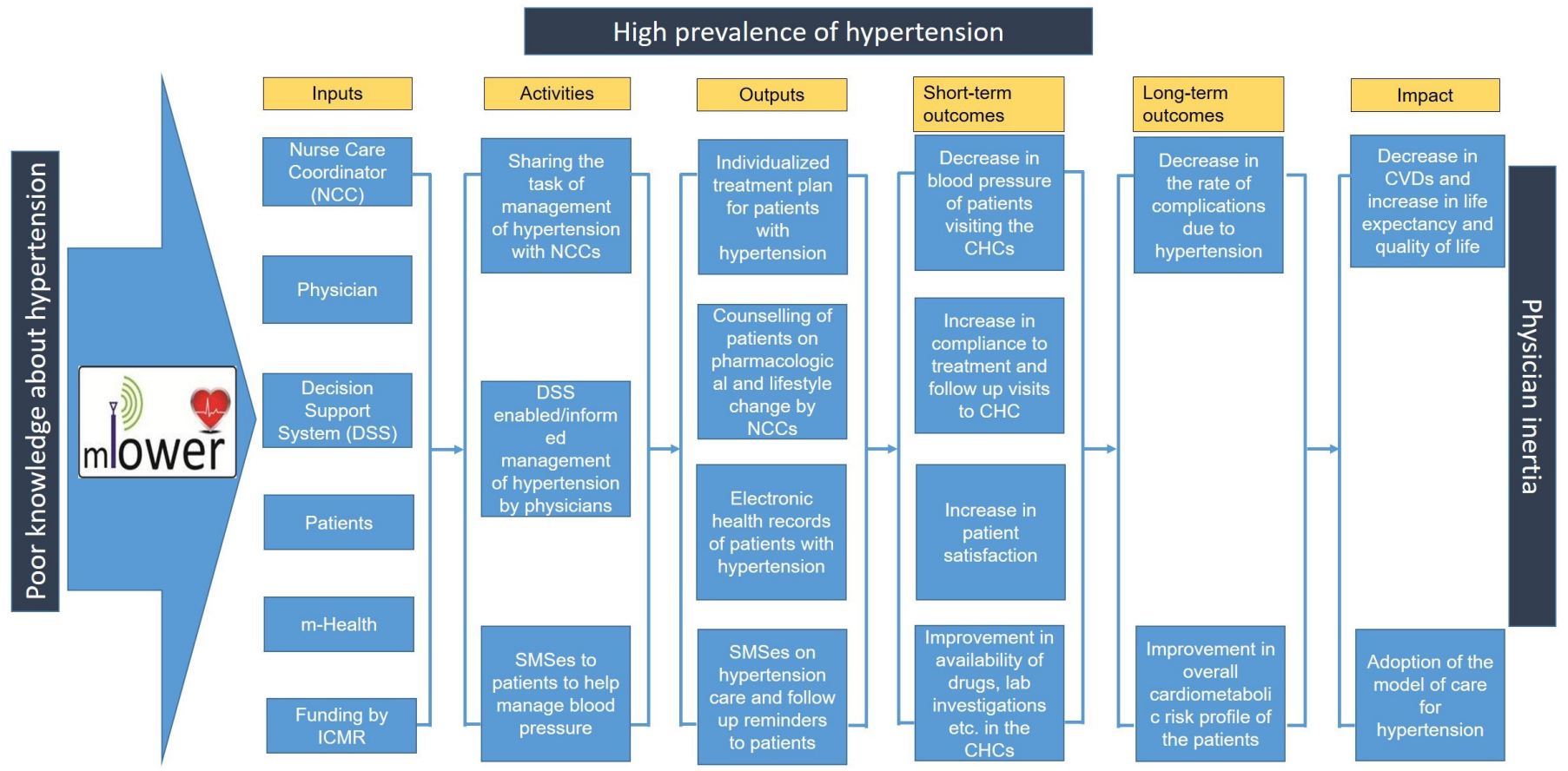

Patient overload at the public health facilities

Figure 1 The logic model of the m-Power Heart Project. CHC, community health centre; DSS, decision support system; ICMR, Indian Council of Medical Research; NCC, nurse care coordinator; SMS, short messaging service.

enabled intervention to decrease blood pressure among patients diagnosed with uncontrolled hypertension (blood pressure $\geq 160 / 90 \mathrm{~mm} \mathrm{Hg}$ ) visiting the community health centres (CHCs) in India. Full details of the trial are published elsewhere ${ }^{7}$; a brief summary of the trial methodology is outlined below. Figure 1 presents the m-Power's logic model.

The m-Power intervention has three main components-an electronic decision support system (EDSS), short messaging service (SMS) and trained NCCs. The EDSS is a hypertension treatment algorithm incorporating national and international guidelines on hypertension management ${ }^{8-10}$ and designed as an android application. The EDSS considers a patient's age, blood pressure, medication use (type, dose and frequency) and co-morbid conditions influencing treatment choices (diabetes, asthma and so on), to generate a suitable treatment plan, which the doctor can use to prescribe treatment. The NCC co-manages the care of hypertensive patients with the doctor. A SMS programme provides information on high blood pressure to patients (risk factors, symptoms, complications, need for regular follow-up and adherence to recommended medications) and reminders for subsequent $\mathrm{CHC}$ visits. The primary outcome is the difference in the mean systolic blood pressure between the intervention and usual care arms at 12 months. The study is designed to detect a $6.5 \mathrm{~mm} \mathrm{Hg}$ systolic blood pressure difference with a power of $80 \%$. Randomisation is at the level of the $\mathrm{CHC}$, allocated at a 1:1 ratio. Each of the 12 participating CHCs will recruit 156 participants to achieve the total sample size of 1872 .

\section{Process evaluation}

RCTs answer the question 'Does the intervention work?' whereas process evaluation of RCTs help to the 'assess fidelity and quality of implementation, clarify the causal mechanisms and identify the contextual factors associated with variation in the outcome/s' ${ }^{11}$ If a trial produces a null effect, process evaluation can differentiate intervention failure from implementation failure. In a multi-centre trial, a process evaluation is necessary to understand if the intervention was implemented and received similarly across all sites. ${ }^{12}$ Context plays a crucial role in either the success or failure of an intervention. Process evaluation aids in the understanding of contextual factors associated with intervention and implementation effectiveness, which can influence potential replication in different settings as well as scale up. Process evaluation often involves ad hoc selection of factors that may be important for understanding a trial's implementation. ${ }^{13}$ Although this approach is better than the absence of any evaluation, a theory-informed process evaluation is better placed to understand the full determinants of outcomes. As such, theory-informed process evaluation findings may be more generalisable to similar problems in similar contexts among similar groups. ${ }^{13}$

We propose to conduct process evaluation of m-Power, guided by the Consolidated Framework for Implementation Research (CFIR) ${ }^{14}$ to understand 'what works, where, why and under what context?' CFIR is a systematic approach to identify factors that influence intervention implementation and effectiveness. The framework consists of five domains (intervention characteristics, 


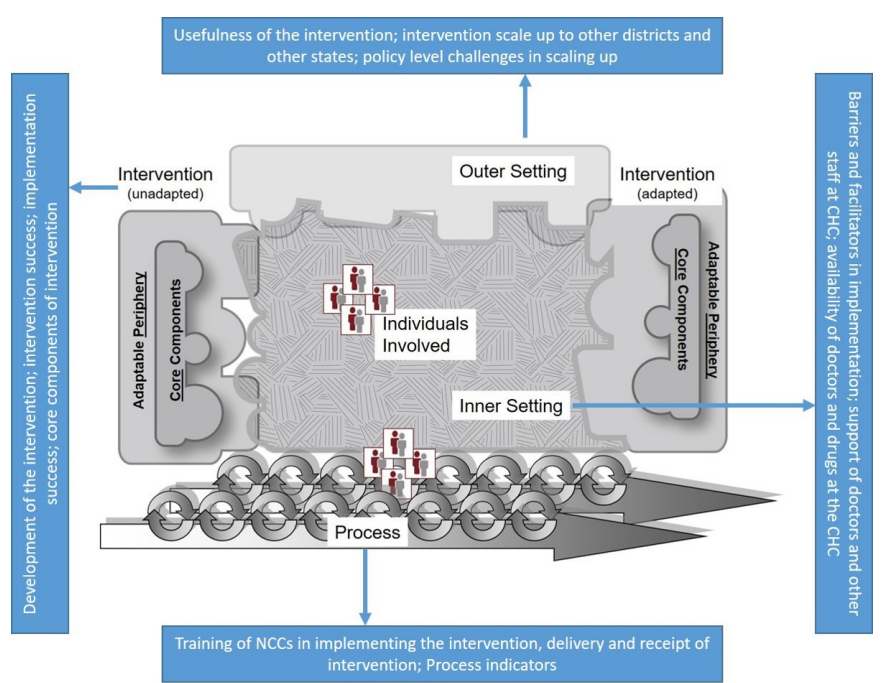

Figure 2 Select process evaluation components in relation to the Consolidated Framework for Implementation Research domains. $\mathrm{CHC}$, community health centre; NCC, nurse care coordinator.

outer setting, inner setting, characteristics of individuals and process). ${ }^{14}$ The CFIR can be used to understand why implementation may or may not succeed, and identify potential barriers and facilitators to implementation. ${ }^{15} \mathrm{We}$ seek answers to the questions below: (1) Who delivers the m-Power intervention and how often? (2) To what extent is m-Power intervention being implemented as planned? (3) How is m-Power intervention received by the target patient group and the CHC staff (doctors, nurses)? (4) What are the barriers to m-Power intervention delivery? and (5) Will any changes be made to the intervention implementation and what are they?

The objectives of the process evaluation are to:

1. Understand the intervention characteristics from the perspectives of different stakeholders-intervention developers, NCCs, doctors, patients and their caregivers.

2. Describe and understand the context under which the intervention is being implemented.

3. Assess the fidelity of implementation.

\section{METHODS AND ANALYSIS}

A mixed-methods process evaluation of m-Power will be carried out using a concurrent triangulation design. The purpose of the mixed-methods is to corroborate the effectiveness results (convergence) and to gain insights about the intervention, implementation and context, which can offset the inherent limitations, experienced using the quantitative or qualitative methods alone.

\section{Study setting}

The m-Power Heart Project will be implemented in Visakhapatnam, the fourth largest district in the southern Indian state of Andhra Pradesh with a population of 4 200000 people. Visakhapatnam has three distinct geographical areas-urban, rural and tribal. There are
13 CHCs in Visakhapatnam. Within each CHC, three to eight doctors are posted and, on any given day between two to eight doctors are available for patient care. Outpatient clinics run from 09:00 to 13:00 6 days a week with only emergency services provided outside those hours. A comprehensive diabetes and hypertension prevention and management programme entitled $\mathrm{UDAY}^{16}$ is currently being implemented in one of the CHC and so it was excluded from m-Power.

\section{Theoretical approach}

The CFIR was chosen to evaluate implementation of the m-Power Heart Project, as it is a complex intervention being implemented in a healthcare setting. The CFIR provides a degree of flexibility wherein researchers can select the constructs that are the most relevant to the study setting and use these to assess context, implementation and explain the study findings. ${ }^{14}$ Given that this process evaluation will not be measuring individual-level behaviour change, the CFIR domain "characteristics of individuals' will not be assessed. Figure 2 presents the process evaluation constructs and their relation to four CFIR domains included in this study.

\section{Study participants}

Participants included in this study represent key stakeholders involved in the m-Power intervention development and implementation. Participants include the m-Power cluster RCT investigators (intervention developers), NCCs (intervention implementers), doctors working in the CHCs (intervention users), patients with hypertension visiting the CHCs and their caregivers (intervention recipients) and health department officials (policy actors). We aim to recruit all $4 \mathrm{~m}$-Power investigators (1 principal and 3 co-investigators); 2 doctors from each intervention CHC; all 6 NCCs (1 from each intervention $\mathrm{CHC}$ ); 8-12 patients, and their caregivers (within each intervention $\mathrm{CHC}$ ); 1 state and 1 district programme officer of the National Programme for Prevention and Control of Cancer, Diabetes, Cardiovascular Disease and Stroke (NPCDCS) and 1 District Coordinator of Hospital Services (in-charge of all CHCs in Visakhapatnam district).

The m-Power investigators will be informed of the process evaluation and invited to take part in an interview via email from an investigator on the process evaluation study (SM). The recruitment email will contain a participant information sheet and consent form. On consent, the first author (NSV) will contact the investigators via email and arrange an interview (either via telephone or in-person) at a time convenient for the investigator. The NCCs, doctors, patients together with their caregivers and health system officials will be recruited by the research assistant employed under the m-Power. The research assistant will meet participants in-person, inform them about the process evaluation and provide individual participant information sheets and consent forms. We expect some patients will be illiterate or inadequately literate, and 
Table 1 Summary of in-depth interviews and focus groups

\begin{tabular}{|c|c|c|}
\hline $\begin{array}{l}\text { Data collection } \\
\text { method }\end{array}$ & Participants & Place of data collection and mode \\
\hline \multirow[t]{3}{*}{ In-depth interviews } & Intervention developers $(n=4)$ & $\begin{array}{l}\text { In-person or via teleconference; investigators } \\
\text { office in Gurgaon, India }\end{array}$ \\
\hline & Doctors $(n=2)$ & In-person; in the CHCs where they are working \\
\hline & $\operatorname{NCCs}(n=6)$ & In-person; in the CHCs where they are working \\
\hline \multirow[t]{2}{*}{ Focus groups } & Patients $(n=12)$ & In the $\mathrm{CHC}$ which they visit for treatment \\
\hline & Patients together with caregivers $(n=12)$ & \\
\hline
\end{tabular}

$\mathrm{CHC}$, community health centre; NCC, nurse care coordinator; NPCDCS, National Programme for Prevention and Control of Cancer, Diabetes, Cardiovascular Disease and Stroke.

for them the information sheet and consent form will be read aloud. If the participants consent to participate, then the research assistant will schedule the interview at a time convenient for the participant, either in the CHC (NCC, doctors and patients with their caregivers) or in the office of the health official.

\section{Data collection}

Qualitative data will contribute to understanding the context, characteristics of the intervention from the perspective of different stakeholders and the challenges of implementation. Quantitative data will be used to assess the key process evaluation indicators. Data collection procedures are summarised in tables 1 and 2 .

\section{In-depth interviews}

In-depth interviews will be conducted with the m-Power intervention developers, doctors, NCCs and health department officials by the first author (NSV). All interviews will be conducted in English with the exception of the interview with NCCs, who will be interviewed in the local language Telugu (spoken by all NCCs). After informing the participants about the study objectives and procedure, a series of open-ended questions will be asked to elicit opinions on the intervention development, intervention implementation challenges, facilitating and inhibiting factors, factors crucial for implementation, intervention effectiveness and potential scale up. Questions will capture participants' understanding and perceptions of the intervention, context and process of implementation.

\section{Focus group discussions}

Two sets of focus groups will be conducted in each intervention CHC-(a) among patients with hypertension and (b) among patients with hypertension together with their caregivers. A total of 24 focus groups will be conducted by the first author, with each focus group expected to last up to 1.5 hours. Each focus group will consist of 8-12 participants who will be purposively sampled to ensure adequate representation from different groups. Focus groups will elicit participant's experience of receiving the intervention, barriers and facilitators in accessing CHC services,

\begin{tabular}{|c|c|c|}
\hline Data type & Source & Mode of collection \\
\hline $\begin{array}{l}\text { Process indicators } \\
\text { Number of patients recruited and retained } \\
\text { throughout the intervention. } \\
\text { Number of visits by the patients. } \\
\text { Number of treatment plans generated. } \\
\text { Concordance between the doctor and } \\
\text { EDSS generated treatment plan. } \\
\text { Number of SMSs sent to patients. } \\
\text { Number of SMSs bounced back. }\end{array}$ & Stored in the m-Power central server & $\begin{array}{l}\text { Captured automatically by the EDSS and } \\
\text { transferred to central server }\end{array}$ \\
\hline \multicolumn{3}{|l|}{ Implementation fidelity } \\
\hline Training. & & OSCE \\
\hline Delivery. & Observation assessment & Checklist, NCC activity diaries \\
\hline
\end{tabular}

EDSS, electronic decision support system; NCC, nurse care coordinator; OSCE, objective structured clinical examination; SMS, short messaging service. 
availability of essential drugs and issues around adopting healthy lifestyle behaviours to reduce blood pressure and so on. Discussions will be moderated by a facilitator (first author) with a note taker (research assistant) present to ensure that all topics are covered and any relevant interactions are recorded. All interviews and focus groups will be audio recorded for later transcription. To accommodate the local language needs and health literacy of the patient group, focus groups will be conducted in Telugu and later transcribed into English.

\section{NCC training evaluation}

The NCCs will be trained to deliver the intervention by the research assistant. The induction training programme consists of learning standardised processes for measuring blood pressure, weight, height and reviewing the lab reports of the patients with hypertension. NCCs will also be trained to use the EDSS and generate the treatment plan that will be shared with the doctors. The training programme takes 132 hours, of which 12 hours are for the final assessment of competency. Training sessions include presentations, lectures, hands-on exercises using the EDSS, mock field visits and patient interactions. Kirkpatrick's model for training evaluation ${ }^{17}$ will be used to evaluate the NCCs training. Three of the four assessment levels will be evaluated-reaction, learning and behaviour. The first two levels will be assessed by the training evaluation. The objective structured clinical examination (OSCE) assessment method will be used to assess learning. The third level (behaviour) will be assessed as a part of the implementation fidelity. The final level in the model (results) will not be assessed as this level includes parameters such as increased production, improved work quality and reduced turnover, which do not apply to the intervention.

\section{Process evaluation indicators}

The following five indicators will be assessed-(1) number of patients recruited and retained throughout the intervention, (2) number of CHC visits by the patients, (3) number of treatment plans generated, (4) concordance between the treatment plans generated by the doctor and EDSS and (5) number of SMSes sent to patients and the number of SMSes that bounced back. The EDSS is designed to capture the information required to measure all five indicators. The information captured will be stored in a secure central server within the Public Health Foundation of India (PHFI) office in Gurgaon, India.

\section{Fidelity of implementation}

Consistent with Borrelli et $a l \mathrm{~s}^{18}$ framework, fidelity will be assessed according to the following categories-design, training, delivery and receipt. Enactment (also included in Borrelli et al s framework) is being excluded as it is beyond the scope of the current study. The category of training has been explained under the NCC training evaluation section. During the trial implementation, observations in the intervention CHCs will be carried out by the first author at least monthly, and at least fortnightly by the trained research assistant. The observation assessment will last $\sim 30$ min with both assessors completing a structured checklist consisting of items to evaluate the workspace, use of logistics, interaction of the NCC with the patient, eliciting patient history, entering data into the EDSS, measuring blood pressure and generating a treatment plan. NCCs are required to maintain a daily activity diary. A minimum of one session is to be carried out per week by each NCC in their respective CHC. The number of counselling sessions conducted and number of patients attending each session will be extracted from the NCC activity diaries. Participant exit interviews after a counselling session with the NCC will assess receipt of the intervention. An interviewer administered questionnaire will be developed in English, translated into Telugu and back translated to English to check for consistency. The interview will be administered by the research assistant in Telugu to gather information about the counselling session vis-à-vis quality, recall of the key messages and satisfaction levels. A total of 25 participants ( 5 each from 5 different sessions spread over 6 months) from each of the intervention CHC will be selected randomly and interviewed, yielding a total of 90 interviews.

\section{Analysis}

The interview transcripts will be translated (from Telugu to English) and transcribed as soon as the interviews are completed to allow for changes in the interview and discussion guide, if required. Audio recordings will be transcribed and translated to English as soon as possible after interviews and focus groups. A single investigator (NSV) will perform all transcription and translation for consistency purposes. Data immersion, coding, category creation and thematic analysis will be undertaken by a single investigator (NSV) using the template analysis technique $^{19}$ for the coding steps. This technique uses predefined codes, which may be amended, or new codes added based on the transcription data. Transcripts will be entered into NVivo V.11 for analysis. Ten transcripts will be independently analysed by a researcher based at PHFI but not associated with the m-Power, to establish inter-rater reliability. Discrepancies will be discussed with the study co-authors (KMN or HK) until consensus is reached that the coding is representative of the data.

The NCC training evaluation data from the OSCE will be summarised into medians (IQR) and the change in scores pre-training and post-training will be calculated. The checklist data will be summarised into means and a mean score will be calculated. The number of counselling sessions and the participation in each session will be enumerated and totalled. The proportion of study participants attending at least $80 \%$ of the sessions will be calculated. Means and proportions will be calculated for the data from the exit interviews. All data analysis will be carried out in Stata V.14.

For the mixed-methods analysis, apart from the qualitative and quantitative data described above, we will use the 


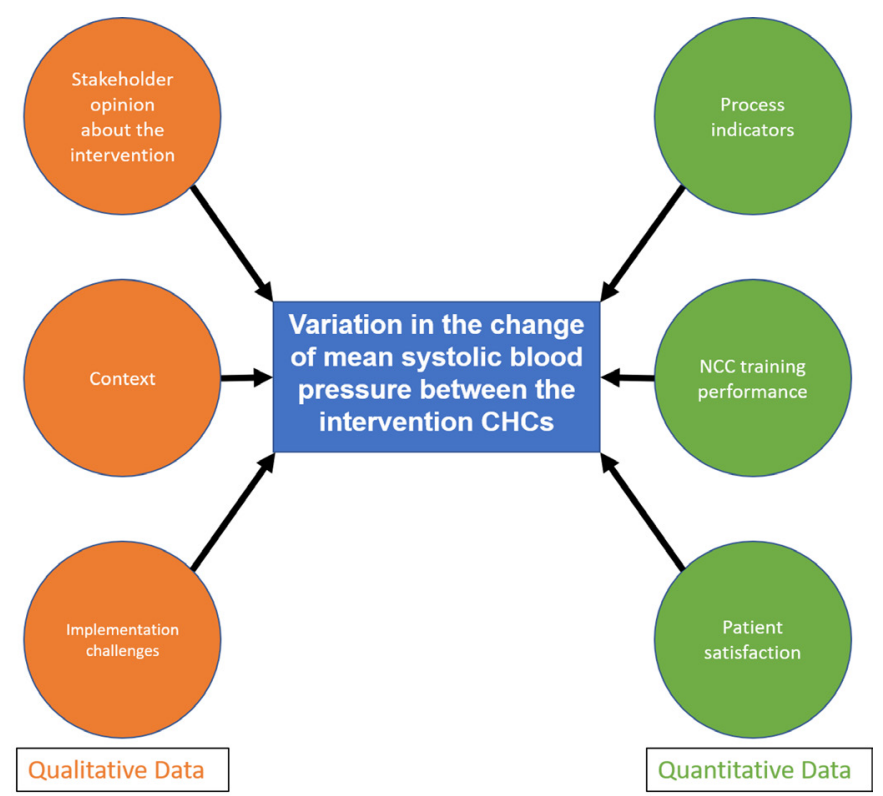

Figure 3 Schema of mixed-methods analysis design. $\mathrm{CHCs}$, community health centres; NCC, nurse care coordinator.

result of primary outcome of the trial for each intervention CHC (mean change in the systolic blood pressure). We will look for convergence by analysing the qualitative and quantitative data to explore if the variation in the mean reduction of systolic blood pressure between the intervention CHCs is influenced by factors like patient satisfaction, session attendance, training outcome, attitude of doctors, patients and NCCs about the intervention, process indicators and so on (figure 3 ).

\section{Patient and public involvement}

The process evaluation is comprehensive as we plan to include all stakeholders. Patients and their caregivers are study participants. We will elicit information on receipt of intervention, challenges in implementing lifestyle changes to control blood pressure, barriers to access services at the CHC and so on. The patients will also be interviewed as a part of exit interview to assess the receipt and recall of key messages, satisfaction level with the NCC sessions and so on. The results of the study will be shared with the patients and their caregivers by providing a one-page summary in local language through the NCCs. In the manuscript, patients and their caregivers will be acknowledged for their contribution.

\section{ETHICS AND DISSEMINATION}

Findings will be disseminated via peer-reviewed publications, national and international conference presentations.

\section{CONCLUSION}

This study will be the first theory-based process evaluation of a hypertension management trial in India.
Although studies have been conducted in India to test the efficacy of task shifting, EDSS and mHealth on blood pressure control in hypertension, either alone or in combination, ${ }^{20-22}$ none of them were assessed using a rigorous process evaluation. To tackle the burden of hypertension, innovative approaches are required and we believe that the m-Power Heart Project intervention is one such solution. Our process evaluation is guided by the use of an evidence-based framework designed to evaluate healthcare innovations, and it will be conducted alongside the intervention implementation thus giving information about the early implementation phase when challenges often occur. We do anticipate following challenges in conducting the evaluation-low literacy among some patients (and possibly their care givers), hierarchical culture and social desirability bias. Findings from this process evaluation will elucidate which contextual factors are associated with intervention effectiveness and the challenges to implementation within the public healthcare system of India. Findings will also inform the wider implementation of the intervention through the NPCDCS and the potential scale up in other states in India. If the trial findings are positive, the evaluation can provide evidence to embed the intervention into the aforesaid existing national programme to address hypertension control strategies. If the trial findings are negative, then the evaluation will help understand whether it was an intervention or implementation failure.

\section{Author affiliations}

${ }^{1}$ School of Exercise and Nutrition Sciences, Deakin University, Burwood, Victoria, Australia

${ }^{2}$ Centre for Chronic Conditions and Injuries, Public Health Foundation of India, Gurgaon, Haryana, India

${ }^{3}$ School of Medicine, Deakin University, Burwood, Victoria, Australia

${ }^{4}$ Centre for Chronic Disease Control, New Delhi, Delhi, India

${ }^{5}$ Interventional Cardiology, KIMS ICON Hospital, Visakhapatnam, Andhra Pradesh, India

${ }^{6}$ Department of Cardiology, All India Institute of Medical Sciences, New Delhi, Delhi, India

${ }^{7}$ Department of Endocrinology and Metabolism, All India Institute of Medical Sciences, New Delhi, Delhi, India

Contributors NSV conceptualised the process evaluation and prepared the first draft of the manuscript. KMN, HK, SM, TW, RM and SO provided critical inputs on the methodology. VSA, DJ, BRM, AR, NT and DP contributed to the development electronic decision support system algorithm. VSA, DJ, NT and DP acquired the funding from Indian Council for Medical Research. All authors read and approved the final version of the manuscript.

Funding $\mathrm{m}$-Power Heart Project is funded by the Indian Council of Medical Research (grant number-DHR/5/GIA/2015-16). The funding agency has no role in the design of the study or in the collection, analysis and interpretation of data or in writing the manuscript. NSV has been awarded a scholarship by Deakin University to pursue PhD under Public Health Foundation of India—Deakin India Research Initiative to conduct the study.

Competing interests None declared.

Patient consent for publication Not required.

Ethics approval Ethical approval for this study was obtained from the ethics committees at Public Health Foundation of India (TRC-IEC-362/17.1) and Deakin University (2018-020). The m-Power trial ethical approval was obtained from the Centre for Chronic Disease Control, Delhi ethics committee (CCDC_IEC_01_2017).

Provenance and peer review Not commissioned; externally peer reviewed. 
Open access This is an open access article distributed in accordance with the Creative Commons Attribution Non Commercial (CC BY-NC 4.0) license, which permits others to distribute, remix, adapt, build upon this work non-commercially, and license their derivative works on different terms, provided the original work is properly cited, appropriate credit is given, any changes made indicated, and the use is non-commercial. See: http://creativecommons.org/licenses/by-nc/4.0/.

\section{REFERENCES}

1. Vital Statistics Division, Ministry of Home Affairs, Government of India. Office of the registrar general india. report on medical certification of cause of death. New Delhi: Vital Statistics Division, Ministry of Home Affairs, Government of India, 2013.

2. Dandona L, Dandona R, Kumar GA, et al. Nations within a nation variations in epidemiological transition across the states of India, 1990-2016 in the global burden of disease study. The Lancet 2017;390:2437-60.

3. Prabhakaran D, Jeemon P, Roy A. Cardiovascular diseases in India: current epidemiology and future directions. Circulation 2016;133:1605-20.

4. Prabhakaran D, Jeemon $P$, Sharma $M$, et al. The changing patterns of cardiovascular diseases and their risk factors in the states of India: the Global Burden of Disease Study 1990-2016. Lancet Glob Health 2018;6:e1339-51.

5. Geldsetzer P, Manne-Goehler J, Theilmann M, et al. Diabetes and hypertension in India: a nationally representative study of 1.3 million adults. JAMA Intern Med 2018;178:363-72.

6. Anchala R, Kannuri NK, Pant $\mathrm{H}$, et al. Hypertension in India: a systematic review and meta-analysis of prevalence, awareness, and control of hypertension. J Hypertens 2014;32:1170-7.

7. Srinivasapura Venkateshmurthy N, Ajay VS, Mohan S, et al. m-power heart project - a nurse care coordinator led, mhealth enabled intervention to improve the management of hypertension in India: study protocol for a cluster randomized trial. Trials 2018;19:429.

8. WHO. Guidelines for assessment and management of cardiovascular risk. Geneva, Switzerland: World Health Organization, 2007.

9. James PA, Oparil S, Carter BL, et al. evidence-based guideline for the management of high blood pressure in adults: report from the panel members appointed to the Eighth Joint National Committee (JNC 8). JAMA 2014;2014:507-20.

10. Mancia G, Fagard R, Narkiewicz K, et al. ESH/ESC guidelines for the management of arterial hypertension: the Task Force for the Management of Arterial Hypertension of the European Society of
Hypertension (ESH) and of the European Society of Cardiology (ESC). Eur Heart J 2013;2013:2159-219.

11. Craig P, Dieppe P, Macintyre S, et al. Developing and evaluating complex interventions: the new Medical Research Council guidance. BMJ 2008;337:a1655

12. Oakley A, Strange V, Bonell C, et al. Process evaluation in randomised controlled trials of complex interventions. BMJ 2006;332:413-6.

13. Presseau J, Grimshaw JM, Tetroe JM, et al. A theory-based process evaluation alongside a randomised controlled trial of printed educational messages to increase primary care physicians' prescription of thiazide diuretics for hypertension [ISRCTN72772651] Implement Sci 2016;11:121.

14. Damschroder LJ, Aron DC, Keith RE, et al. Fostering implementation of health services research findings into practice: a consolidated framework for advancing implementation science. Implement Sci 2009;4:50.

15. Breimaier HE, Heckemann B, Halfens RJ, et al. The consolidated framework for implementation research (cfir): a useful theoretical framework for guiding and evaluating a guideline implementation process in a hospital-based nursing practice. BMC Nurs 2015;14:43.

16. Mohan S, Jarhyan P, Ghosh S, et al. UDAY: A comprehensive diabetes and hypertension prevention and management program in India. BMJ Open 2018;8:e015919.

17. Kirkpatrick DL, Kirkpatrick JD. Evaluating training programs the four levels. San Francisco, USA: Berrett - Koehler Publishers, 2010.

18. Borrelli B, Sepinwall D, Ernst D, et al. A new tool to assess treatment fidelity and evaluation of treatment fidelity across 10 years of health behavior research. J Consult Clin Psychol 2005;73:852-60.

19. King N. "Using templates in the thematic analysis of text", in $C$ Cassell \& G Symon, Essential guide to qualitative methods in organizational research. London, UK: SAGE Publications Ltd, 2004.

20. Anchala R, Kaptoge S, Pant H, et al. Evaluation of effectiveness and cost-effectiveness of a clinical decision support system in managing hypertension in resource constrained primary health care settings: results from a cluster randomized trial. J Am Heart Assoc 2015;4:e001213.

21. Tian M, Ajay VS, Dunzhu D, et al. A cluster-randomized, controlled trial of a simplified multifaceted management program for individuals at high cardiovascular risk (simcard trial) in rural tibet, China, and Haryana, India. Circulation 2015;132:815-24.

22. Ajay VS, Jindal D, Roy A, et al. Development of a smartphoneenabled hypertension and diabetes mellitus management package to facilitate evidence-based care delivery in primary healthcare facilities in India: the mpower heart project. J Am Heart Assoc 2016;5. 